\title{
Proton Form Factor Measurements at Jefferson Lab
}

\author{
C.F. Perdrisat ${ }^{1 \dagger}$, V. Punjabi ${ }^{2}$ and the Jefferson Lab $G_{E p}(\mathrm{III})$ Collaborations $^{3}$ \\ (1) the College of William and Mary, Williamsburg, VA 23187 \\ (2) Norfolk State University, Norfolk, VA 23504 \\ (3) 12000 Jefferson Avenue, Newport News, VA 23666, USA \\ $\dagger$ E-mail: perdrisa@jlab.org
}

\begin{abstract}
In two experiments at Jefferson Lab in Hall A, the first one in 1998 and the second in 2000 , the ratio of the electromagnetic form factors of the proton was obtained by measuring $P_{t}$ and $P_{\ell}$, the transverse and longitudinal recoil proton polarization components, respectively, in $\vec{e} p \rightarrow e \vec{p}$; the ratio $G_{E p} / G_{M p}$ is proportional to $P_{t} / P_{\ell}$. Simultaneous measurement of $P_{t}$ and $P_{\ell}$ provides good control of the systematic uncertainty. The first measurement of $G_{E p} / G_{M p}$ ratio was made to $Q^{2}=3.5 \mathrm{GeV}^{2}$ and the second measurement to $Q^{2}=5.6 \mathrm{GeV}^{2}$. The results from these two experiments indicate that the ratio scales like $1 / Q^{2}$, in stark contrast with cross section data analyzed by the Rosenbluth separation method which gives a constant value for this ratio. The incompatibility of the recoil polarization results with most of the Rosenbluth separation results appears now well established above $\mathrm{Q}^{2}$ of about $3 \mathrm{GeV}^{2}$. The consensus at the present time is that the interference of the two-photon exchange with the Born term, which had been deemed negligible until recently, might explain the discrepancy between the results of the two techniques; the possibility that the discrepancy is due to incomplete radiative correction has also been recently discussed.
\end{abstract}

\section{Introduction}

The ratio of the elastic electromagnetic form factors of the proton, $G_{E p} / G_{M p}$, has been measured at Jefferson Lab (JLab) for $\mathrm{Q}^{2}$ values ranging from 0.5 to $5.6 \mathrm{GeV}^{2}$. The technique used was the determination of the recoil proton polarization components in the $\vec{e} p \rightarrow e \vec{p}$ reaction, with both final state particles detected. The results of the two JLab experiments, the first one reaching to $\mathrm{Q}^{2}=3.5 \mathrm{GeV}^{2}$, and the second one to $\mathrm{Q}^{2}=5.6 \mathrm{GeV}^{2}$, are now published $[1,2]$ and well known. The ratio $\mu G_{E p} / G_{M p}$ was found to decrease linearly with $Q^{2}$, from $\approx 1$ at $\mathrm{Q}^{2}=0.5 \mathrm{GeV}^{2}$, down to 0.28 at $Q^{2}=5.6 \mathrm{GeV}^{2}$; in the dipole model this ratio would be 1.0 for all $Q^{2}$ values. These results demonstrated unambiguously for the first time that the $Q^{2}$ dependences of $G_{E p}$ and $G_{M p}$ are different from one another.

Double spin experiments $[3,4]$ measure the product $G_{E p} G_{M p}$ as well as $G_{M p}^{2}$, and hence determine the relative sign of the form factors. The combined results of the two JLab experiments were surprising as they appeared to contradict the consensus based on Rosenbluth separation results for $G_{E p}^{2}$ and $G_{M p}^{2}$ : the ratio $\mu_{p} G_{E p} / G_{M p}$ obtained with 
the Rosenbluth method appear to be near 1 up to $5 \mathrm{GeV}^{2}$ [5]. This un-bridgeable difference between cross section and polarization experiments has been reinforced with two recent JLab Rosenbluth experiments [6, 7]; it appears increasingly difficult to explain the difference in the ratio by methodological or systematic errors.

Cross section data require very large radiative corrections, polarization data do not ([8]). It is still possible that the difference will be explained after reexamination of the standard radiative correction procedure [9]. Another possibility is that the single-photon exchange or Born approximation at the basis of form factor extraction, needs to be reexamined and the effect of two-photon exchange calculated [10]. The preliminary results of such calculations $[11,12]$ indicate that two-photon exchange, which enters as an interference between the one- and two-photon diagrams, can indeed affect the form factor extraction from cross section data significantly, but modifies the polarization results very little. The form factor extraction procedure is based on dominance of one-photon exchange; the consequences of a measurable two-photon exchange contribution for the extraction of form factors have been discussed by [13].

We are currently preparing a third measurement of the $G_{E p} / G_{M p}$-ratio at JLab, to extend the $\mathrm{Q}^{2}$-range to $9 \mathrm{GeV}^{2}$ [14]. This high priority experiment requires a new polarimeter to be installed in the focal plane of the high momentum spectrometer in Hall $\mathrm{C}$, and a new large acceptance calorimeter to detect the electron. Both instruments are now in an advanced stage of construction. The experiment will be ready to go on the Hall $\mathrm{C}$ floor in the later part of 2005 .

To resolve the problem of the incompatibility of the $G_{E p} / G_{M p}$-ratios obtained from cross section and polarization data, we have proposed [15] to measure the ratio $G_{E p} / G_{M p}$ as a function of $\epsilon$, the longitudinal polarization of the virtual photon, by the recoil polarization method. This study requires the same instrumentation as the $G_{E p}($ III) experiment, and will hopefully occur immediately after it.

We will also review the results from several recent $G_{E p}$ experiments at JLab, and present the status of $G_{E n}[16,17,18,19]$ and $G_{M n}[20]$ past and future measurements.

\section{Polarization Method}

In the One-Photon Exchange or Born approximation for elastic ep the observables of a recoil polarization experiment are the two components of the proton polarization in the reaction plane, $P_{t}$ and $P_{\ell}$, as follows:

$$
\begin{aligned}
& I_{0} P_{t}=-2 \sqrt{\tau(1+\tau)} G_{E p} G_{M p} \tan \frac{\theta_{e}}{2} \\
& I_{0} P_{\ell}=\frac{1}{M_{p}}\left(E_{\text {beam }}+E_{e}\right) \sqrt{\tau(1+\tau)} G_{M p}^{2} \tan ^{2} \frac{\theta_{e}}{2}
\end{aligned}
$$

$I_{0}$ is proportional to the unpolarized cross section and is given by:

$$
I_{0}=G_{E p}^{2}+\frac{\tau}{\epsilon} G_{M p}^{2}
$$

where $\epsilon^{-1}=1+2(1+\tau) \tan ^{2}\left(\frac{\theta_{e}}{2}\right)$, and $\tau=Q^{2} / 4 M_{p}^{2}$. 
An out-of-plane polarization component, $P_{n}$, can be the result of the interference between the Born term and 2-photon exchange. Such a component is independent of the beam helicity; it is expected to be at the level of 1-2 percent; it is hard to measure.

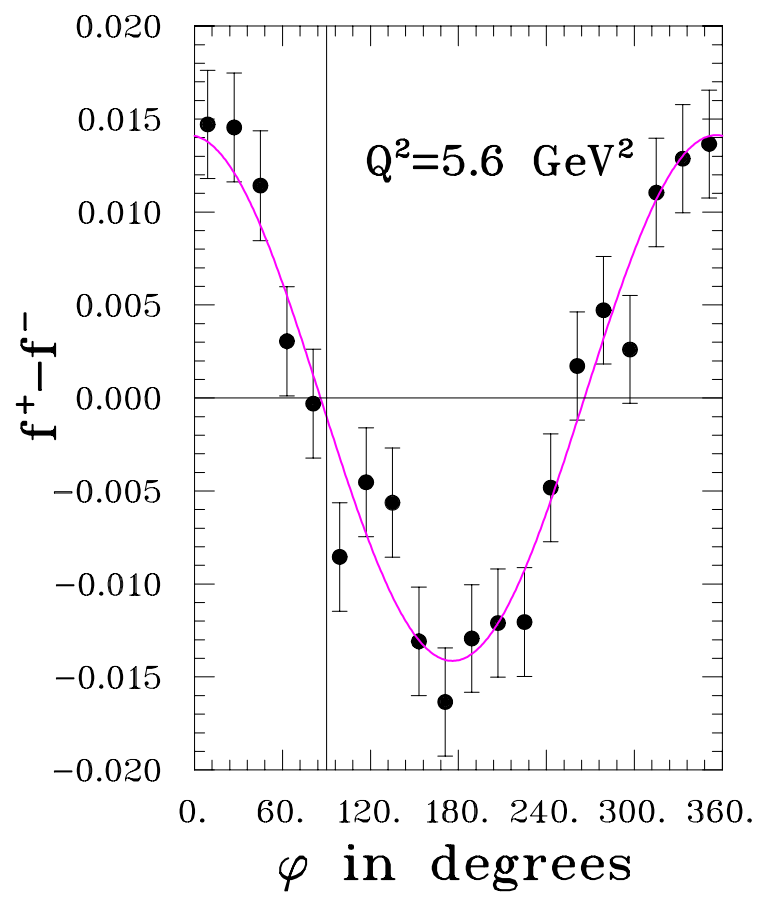

Figure 1: The azimuthal asymmetry distribution for $\mathrm{Q}^{2}=5.6 \mathrm{GeV}^{2}$.

In the two recoil polarization experiments at JLab the polarization of the recoil proton was measured in a polarimeter installed in the focal plane area of one of the large magnetic spectrometers of Hall A. A focal plane polarimeter consists of an analyzer block of $C$ or $\mathrm{CH}_{2}$, preceded and followed by tracking wire chambers, in which the proton is scattered in a nuclear interaction; the normalized angular distribution of the protons scattered in the analyzer is given by:

$$
f^{ \pm}(\varphi)=\frac{1}{2 \pi}\left(1 \pm A_{y} P_{t}^{f p p} \sin \varphi \mp A_{y} P_{n}^{f p p} \cos \varphi\right)
$$

where $P_{n}^{f p p}$ and $P_{t}^{f p p}$ are the physical asymmetries at the focal plane polarimeter, and $A_{y}$ is the analyzing power of the polarimeter; the \pm -sign denotes the two possible helicities of the polarized beam. In the data analysis $f^{ \pm}$is approximated by the differential yield:

$$
\left.Y_{i}^{ \pm}=\frac{1}{\Delta \varphi} \frac{N_{i}^{ \pm}}{N_{i n}^{ \pm} \eta(\vartheta}\right)
$$

where the index $i$ refers to a bin in $\varphi, \Delta \varphi$ is the width of the bin, $N_{i}^{ \pm}$is the number of events in bin $i, N_{i n}^{ \pm}$is the number of protons with specified helicity incident upon the FPP, 
and $\eta(\vartheta)$ is the differential efficiency of the analyzing reaction. Instrumental asymmetries are cancelled by alternating the helicity denoted by \pm of the electron beam, and measuring $Y^{+}$and $Y^{-}$. The difference $Y^{+}-Y^{-}$contains only the helicity dependent polarizations $P_{t}^{f p p}$ and $P_{n}^{f p p}$, whereas the sum $Y^{+}+Y^{-}$contains a possible helicity independent part from two-photon exchange, and the instrumental asymmetries. Fig. 1 shows the azimuthal angular distribution of the asymmetry measured at the highest $\mathrm{Q}^{2}$ reached so far, 5.6 $\mathrm{GeV}^{2}$.

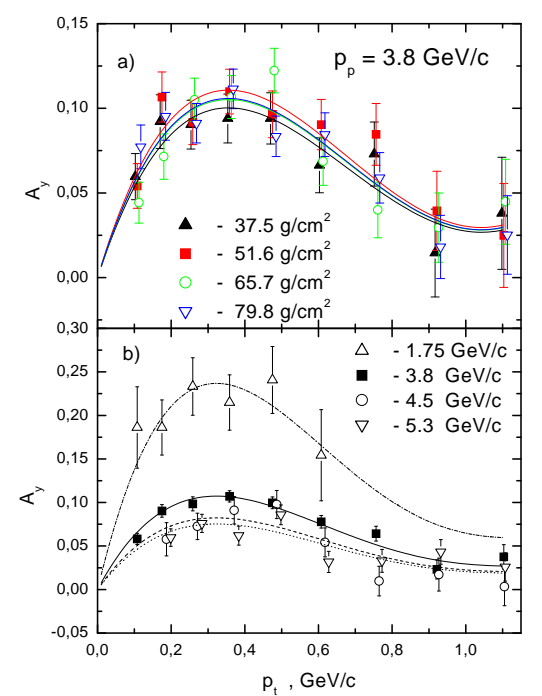

Figure 2: The Dubna calibration results for the analyzing power of $\mathrm{CH}_{2}$ : top panel 4 analyzer thicknesses at $p_{p}=3.8$ $\mathrm{GeV} / \mathrm{c}$. Bottom panel fixed analyzer thickness of $51.6 \mathrm{gcm}^{-2}$ and proton momenta $1.75,3.8,4.5$ and $5.3 \mathrm{GeV} / \mathrm{c}$.

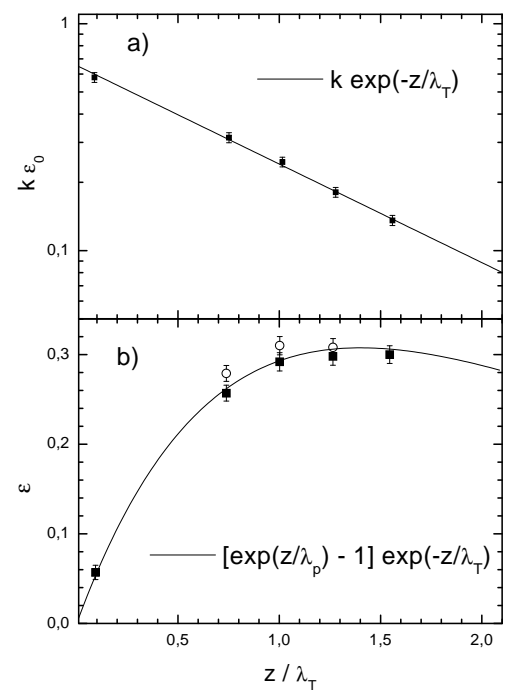

Figure 3: The Dubna calibration results: top panel attenuation of un-scattered beam versus analyzer thickness; bottom panel evolution of the efficiency integral with the analyzer thickness; note that in the text $\epsilon=\eta$.

The polarimeter is characterized by the analyzing power, $A_{y}$, resulting from $\vec{L} . \vec{S}$ coupling in the interaction of the proton with the analyzer nuclei, and the probability of interaction, $\eta(\vartheta)$, which is related to the inclusive differential $\mathrm{pC}$ or $\mathrm{p}\left(\mathrm{CH}_{2}\right)$ yield; $A_{y}$ depends only weakly upon the material of the analyzer, and $\eta(\vartheta)$ is determined by the total mean-free path in the material, $\lambda_{T}$, and the thickness of the analyzer. Hydrogen is best, $\mathrm{CH}_{2}$ next best. Efficiency and analyzing power have been measured in Dubna in preparation for the third $G_{E p}$ JLab experiment [21] and are shown in Figs. 2 and 3.

In the JLab experiments the polarization of the recoil proton is measured in a polarimeter (FPP) located at the focal plane of one of the high resolution magnetic spectrometer. Vertical bending of the trajectories in the spectrometer rotates the polarization component $P_{\ell}$ into a normal component at the analyzer, $P_{n}^{f p p}$, but does not change the polarization component $P_{t}$ in first order.

In first approximation, at the target $P_{\ell}=P_{n}^{f p p} / \sin \chi$, and $P_{t} \sim P_{t}^{f p p}$, where $\chi$ is the spin precession angle in the dispersive plane of the magnetic spectrometer used to detect the recoil proton, given by 


$$
\chi=\gamma \Theta_{B}\left(\mu_{p}-1\right),
$$

where $\Theta_{B}$ is the bend angle of the trajectories in the dipole.

The desired ratio of form factors is then obtained from the target polarization components, $P_{t}$ and $P_{\ell}$, from the relation:

$$
\frac{G_{E p}}{G_{M p}}=-\frac{P_{t}}{P_{\ell}} \frac{E_{e}+E_{e}^{\prime}}{2 m_{p}} \tan \frac{\theta_{e}}{2}
$$

\section{$3 \quad$ Results of previous experiments}

The JLab recoil polarization results are shown as the ratio $\mu_{p} G_{E p} / G_{M p}$, and compared with a selection of Rosenbluth results in Fig. 4. The Rosenbluth data included are those of the last SLAC measurement of Ref. [5], and the new JLab data of Refs. [6, 7].

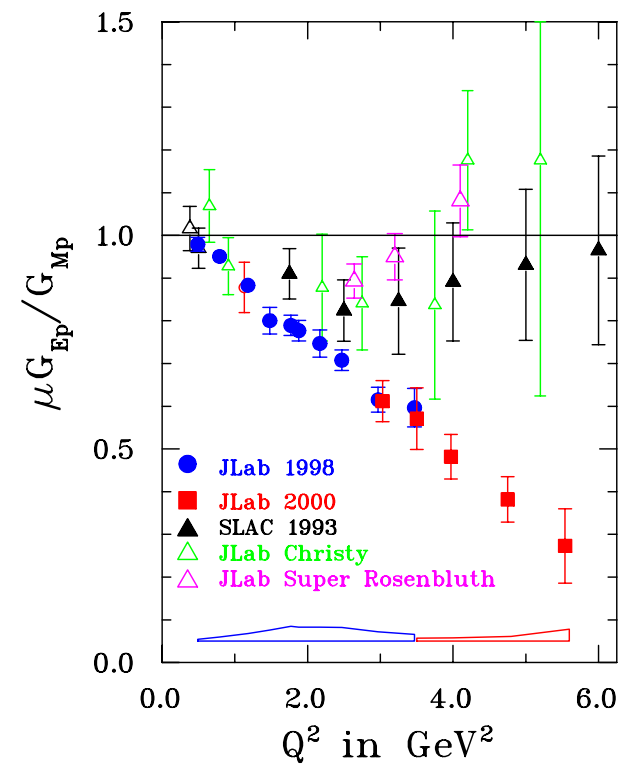

Figure 4: Ratio $\mu_{p} G_{E p} / G_{M p}$ from the $J L a b$ recoil polarization experiments, compared to a selection of ratios obtained by Rosenbluth separation method.

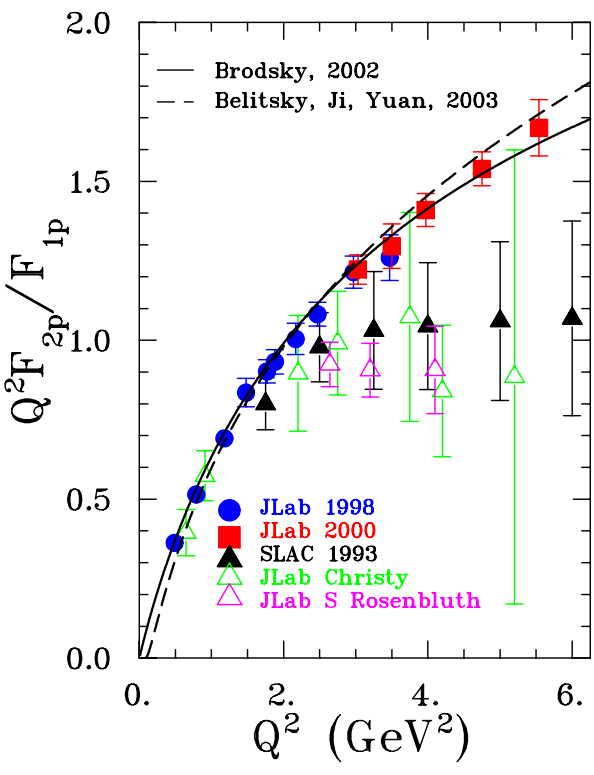

Figure 5: The ratio $Q^{2} F_{2} / F_{1}$ from the JLab recoil polarization experiments, compared to recent $p Q C D$ predictions by Belitsky et al [22] and Brodsky [23].

The Sachs form factor ratio also determine the ratio of the Pauli and Dirac form factors $F_{2} / F_{1}$; the latter are shown in Fig. 5 where they are compared with two recent calculations based on perturbative QCD: the dashed curve is the modified pQCD prediction of Belitsky et al [22], which takes orbital angular momentum of the quarks into account; the solid line is an "empirical fit" from Brodsky [23], taking into account the logarithmic behavior of the $F_{2} / F_{1}$ - ratio inherent in pQCD. 


\section{Theoretical "Predictions"}

Early attempts to understand the nucleon form factors were based on the vector meson dominance model or VMD. In 1972 Iachello [24] had predicted a zero crossing for $G_{E p}$

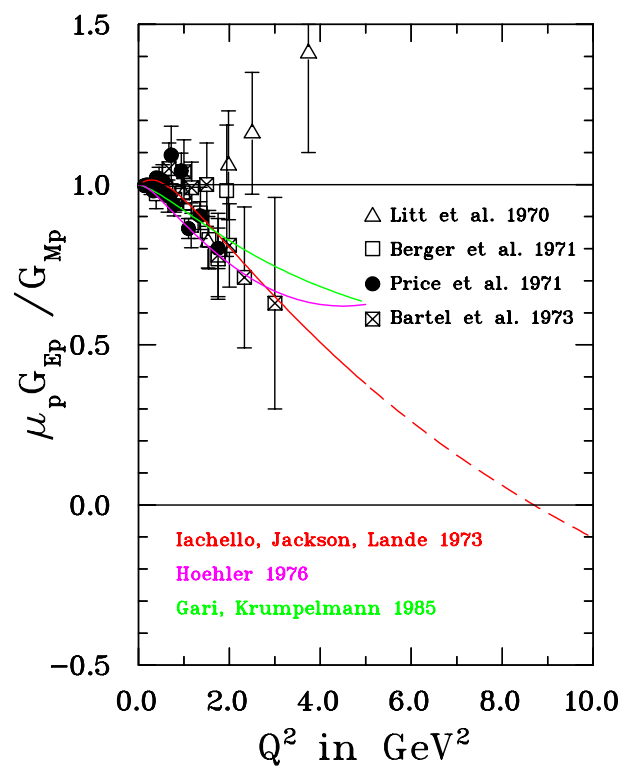

Figure 6: Form factor ratio compared to VMD calculations from the 1970's. Noticeable is Iachello's prediction of a zero crossing of the ratio; his model included a small hard core inside the proton.

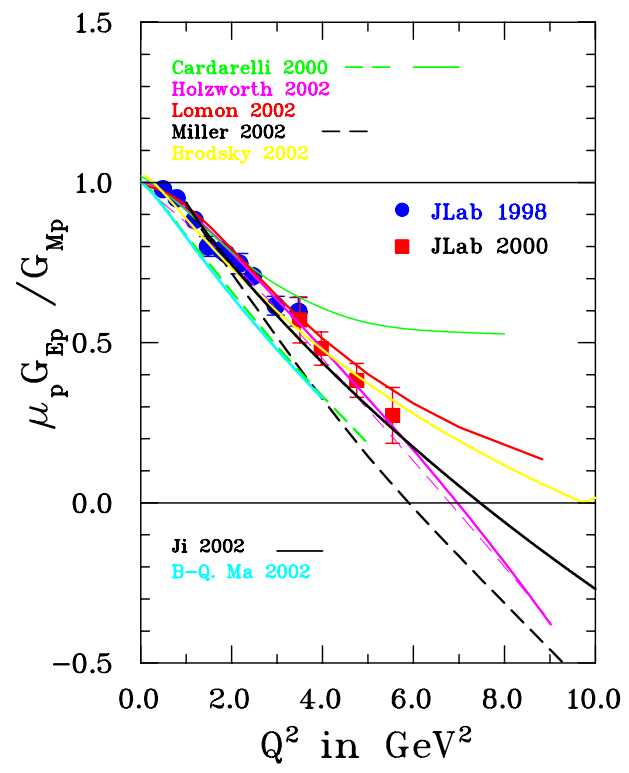

Figure 7: The ratio $Q^{2} F_{2} / F_{1}$ as determined in the recoil polarization experiments 93-027 and 99-007, compared to two recent $p Q C D$ predictions, by $B e-$ litzky et al [22] and Brodsky [23].

near $8 \mathrm{GeV}^{2}$, based on a modified VMD model including a small hard core inside the proton. This prediction is shown in Fig. 6, together with the other VMD fits and data of this period only $[25,26]$. Two years before the first JLab measurement Holzwarth [27] had also predicted a zero crossing of $G_{E p}$, based on the soliton model. This prediction and a number of current versions of the constituent quark [28, 29, 30], VMD [31] and di-quark model [32] predictions are shown in Fig. 7. All forms of the constituent quark model require a relativistic approach to reproduce the recoil polarization data. The relativistic di-quark model of Ma reproduces the right slope of decrease of the $G_{E p} / G_{M p}$ ratio too. The revised pQCD prediction of Brodsky [23] as well as a form of pQCD including quark orbital angular momentum by Belitsky et al are also shown in this figure [22].

\section{Possible origins of the discrepancy}

Radiative corrections (RC) affect $e p$ cross sections at the level of $30-40 \%$ and the data are corrected for it. The correction has to have an accuracy better than or of order $1 \%$ at $\mathrm{Q}^{2}=5.6 \mathrm{GeV}^{2}$ as the contribution of $G_{E p}$ to the cross section is smaller than $10 \%$. The radiative correction to the cross section is $\epsilon$ dependent, and therefore affect the separation result directly; it is based on the seminal papers of Mo and Tsai [33] and Maximon and 
Tjon [34], which do not include the inelastic contribution in the proton vertex correction. Radiative corrections affect the $G_{E p} / G_{M p}$-ratio from polarization data by less than $1 \%$ at a $\mathrm{Q}^{2}$ of $5.6 \mathrm{GeV}^{2}$ when the proton defines the kinematics (see Afanasev [8]).

To explain the discrepancy between Rosenbluth and recoil polarization results, we have to also consider the two-photon exchange contribution; this was recently discussed by Guichon and Vanderhaegen [10], Blunden and Melnitchouk [11], Chen, Afanasev, Brodsky, Carlson and Vanderhaegen [12], and Rekalo and Tomasi-Gustafsson [13].

The possibility that the discrepancy is an experimental effect must of course also be examined. It appears that the discrepancy cannot be due to experimental systematics in the cross section measurement, as the recent "Super" Rosenbluth separation demonstrates (Segel at al [7]). This new JLab experiment was the first ${ }^{1} H(e, p)$ measurement; all previous separations were based on $\left.{ }^{1} H\left(e, e^{\prime}\right)\right)$; in this case radiative corrections are both smaller and different; yet the results are compatible with older Rosenbluth data. They are shown in Fig. 4 as red open triangles.

\section{Two-Photon Exchange Contribution}

Two-photon affects polarization observables and cross sections at the same level of a few $\%$; but at large $\mathrm{Q}^{2}$ the form factors from Rosenbluth separation are strongly affected by this small correction which is $\epsilon$ dependent and $G_{E p}^{2} \ll \tau G_{M p}^{2}$, as we now know from the recoil polarization experiments.

The transverse polarization component, and therefore $G_{E p} / G_{M p}$, is affected only at the level of a few \%; and this is the beauty of the method!

The calculations in Ref. [12] are based on generalized parton distributions which fit the proton form factor. The correction they calculate brings the Rosenbluth form factor results in approximative agreement with the recoil polarization results. Their prediction for the recoil polarization experiments is shown as a 3-dimensional plot in Fig. 8, as the absolute correction $\Delta(\mu R)$ to the form factor ratio $R=\mu_{p} G_{E p} / G_{M p}$, versus $\epsilon$ and $\mathrm{Q}^{2}$. It can be seen in this figure that at $9 \mathrm{GeV}^{2}$, the two-photon correction is smaller than the anticipated statistical uncertainty of 0.08 (see below) by a factor of 3-4.

Two-photon exchange affects form factor observables as an interference between the single- and two-photon processes. As a result the T-matrix depends on three complex amplitudes, $\tilde{G}_{M}, \tilde{F}_{2}$ and $\tilde{F}_{3}$, instead of 2 real and relativistically invariant amplitudes, $G_{M}\left(Q^{2}\right)$ and $G_{E}\left(Q^{2}\right)$, which can be written as [10]:

$$
T=\frac{e^{2}}{Q^{2}} \bar{u}\left(k^{\prime}\right) u(k) \bar{u}_{p}\left(p^{\prime}\right)\left(\tilde{G}_{M} \gamma^{\mu}-\tilde{F}_{2} \frac{P^{\mu}}{M}+\tilde{F}_{3} \frac{\gamma \cdot K P^{\mu}}{M^{2}}\right) u(p)
$$

In Born approximation, the first two amplitudes are the usual $G_{M}$ and $F_{2}$ (real) functions, and $\tilde{F}_{3}=0$. The polarization components are then given as [10]:

$$
\begin{aligned}
P_{t}= & -\sqrt{\frac{2 \epsilon(1-\epsilon)}{\tau}} \frac{C_{B}\left(\epsilon, Q^{2}\right)\left|\tilde{G}_{M}\right|^{2}}{d \sigma} \\
& \times\left\{\frac{\left|\tilde{G}_{M}\right|-\cos \phi_{2 M}\left|\tilde{F}_{2}\right|(1+\tau)}{\left|\tilde{G}_{M}\right|}+\frac{\nu \cos \phi_{3 M}\left|\tilde{F}_{3}\right|}{M^{2}\left|\tilde{G}_{M}\right|}\right\}
\end{aligned}
$$




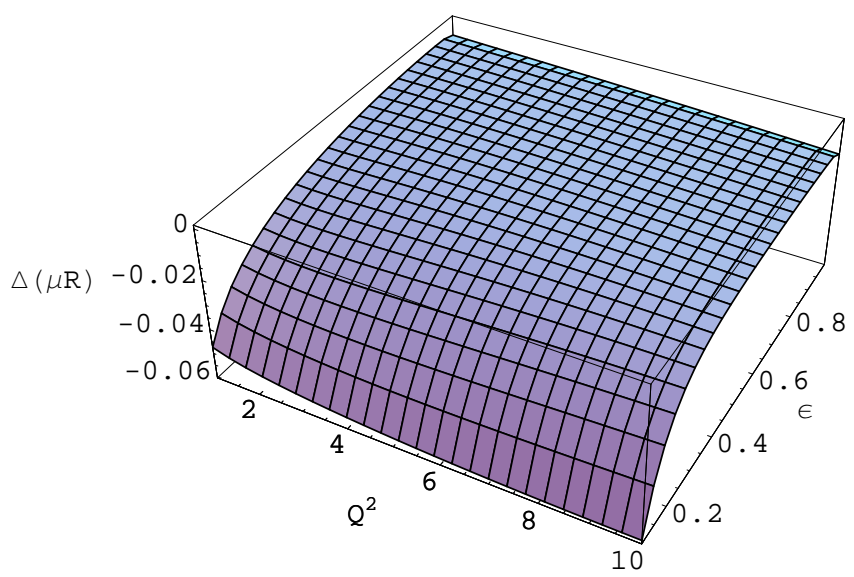

Figure 8: Calculated values of the correction $\Delta \mu_{p} G_{E p} / G_{M p}$ to the measured ratio versus $Q^{2}$ and $\epsilon$.

$$
\begin{aligned}
P_{l}= & \sqrt{(1+\epsilon)(1-\epsilon)} \frac{C_{B}\left(\epsilon, Q^{2}\right)\left|\tilde{G}_{M}\right|^{2}}{d \sigma} \\
& \times\left\{1+\frac{2 \epsilon}{1+\epsilon} \cdot \frac{\nu \cos \phi_{3 M}\left|\tilde{F}_{3}\right|}{M^{2}\left|\tilde{G}_{M}\right|}\right\}
\end{aligned}
$$

where $C_{B}$ is a phase space factor.

One approach consists in connecting measured cross-sections and polarization component ratios at the same $\mathrm{Q}^{2}$ and range of $\epsilon$-values, and extract by a fitting procedure both the unknown "true" form-factor ratio $R$ and the unknown two-photon contribution $Y_{2} \gamma$ :

$$
\begin{array}{r}
\frac{d \sigma_{r e d}(\varepsilon)}{\left|\tilde{G}_{M}\right|^{2}}=1+\frac{\varepsilon}{\tau} R^{2}+2 \varepsilon\left(1+\frac{R}{\tau}\right) Y_{2 \gamma}(\varepsilon) \\
\frac{P_{t}}{P_{l}}\left(\varepsilon_{p}\right)=-\sqrt{\frac{2 \varepsilon_{p}}{\tau\left(1+\varepsilon_{p}\right)}} \frac{R+Y_{2 \gamma}\left(\varepsilon_{p}\right)}{1+2 \varepsilon_{p} Y_{2 \gamma}\left(\varepsilon_{p}\right) /\left(1+\varepsilon_{p}\right)}
\end{array}
$$

where $\varepsilon$ and $\varepsilon_{p}$ denote the $\epsilon$-values at which we will have cross section and polarization data, respectively. The approved experiment in Hall C, 09-019, will measure $G_{E p} / G_{M p}$ at fixed $\mathrm{Q}^{2}$ of $2.6 \mathrm{GeV}^{2}$ with $1 \%$ statistics at 3 values of $\epsilon, 0.12,0.60$ and 0.78 [35].

\section{The next Experiment}

\subsection{New Polarimeter}

The experiment $G_{E p}(\mathrm{III})$ will extend the $\mathrm{Q}^{2}$-range to $9 \mathrm{GeV}^{2}$ and requires a better polarimeter than used in the two previous experiments. It is not possible to increase the 
analyzing power. Increasing the analyzer thickness does not work either; but increasing the number of polarimeters in series does work. We have chosen a configuration for the new polarimeter with two identical FPPs in series, as shown in Fig. 9.

The forthcoming third phase of investigation of the proton form factors could not have

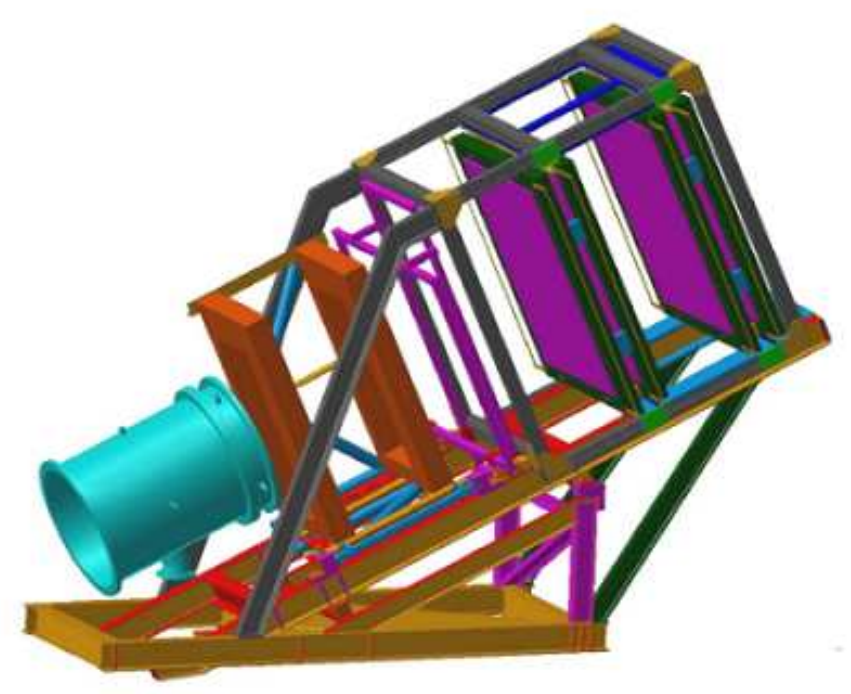

Figure 9: Design drawing of the double polarimeter under construction for the third $G_{E} p$ experiment in Hall C. The orange boxes are the permanent HMS focal plane drift chambers. The two magenta boxes represent the two pairs of drift chamber of the FPP; the two $\mathrm{CH}_{2}$ analyzer blocks are not shown.

started without the $A_{y}$ measurement in Dubna in 2001 [21], shown in Figs. 2 and 3 up to proton momentum of $5.3 \mathrm{GeV} / \mathrm{c}$, with polarized proton beams obtained by breaking up polarized deuterons produced by the Synchrophasotron.

We have also tested the two polarimeter concept in Hall A by reconfigurating the FPP installed in the high resolution hadron spectrometer (HRSh). Data with a first $\mathrm{CH}_{2}$ analyzer following the two focal plane drift chambers, followed by the two front chambers of the FPP, and then the permanent $\mathrm{C}$ analyzer followed by the two FPP back chambers have been taken during the real Compton scattering (RCS) and deuteron two-body photo-desintegration $\gamma d \rightarrow p(n)$. The results are shown in Fig. 10. The first analyzer was $44 \mathrm{~cm}$ of $\mathrm{CH}_{2}$ and the second analyzer $50 \mathrm{~cm}$ of $\mathrm{C}$. The data confirm that $A_{y}$ is larger for $\mathrm{CH}_{2}$ than for $\mathrm{C}$ and that $A_{y}$ is the same for 44 and $100 \mathrm{~cm}$ of $\mathrm{CH}_{2}$ from experiment JLab 99-007. Total efficiency for the double polarimeter was $\sim 50 \%$, even though there were geometrical restrictions in the acceptance of the first polarimeter due to the improvised nature of the test.

The new polarimeter requires 4 large drift chambers; these chambers are currently being built in the Instrumentation group of the Laboratory for High Energy (LHE) at the Joint Institute for Nuclear Research (JINR) in Dubna, under the leadership of Prof. Yu. Zanevsky. A prototype fifth chamber has been received at JLab in November 2003, and is shown in Fig. 11. This is another fine example of the productive collaboration between LHE and our group in Virginia. 

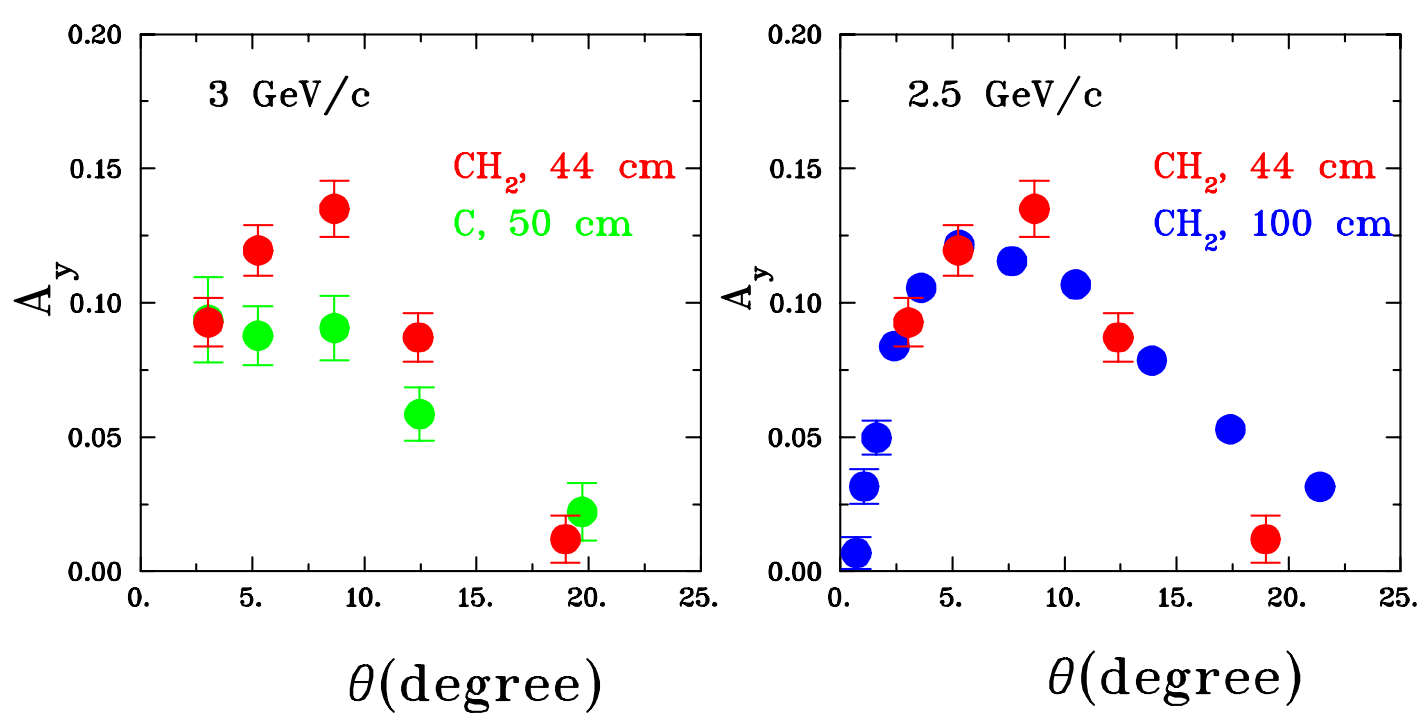

Figure 10: Left panel: analyzing power in the $\mathrm{CH}_{2}$ and $C$-sections of the double polarimeter clearly show the advantage of $\mathrm{CH}_{2}$. Right panel: comparison of the result for $44 \mathrm{~cm}$ of $\mathrm{CH}_{2}$, with those of ref. [2] for $100 \mathrm{~cm}$ of $\mathrm{CH}_{2}$ confirming that no loss of analyzing power is visible with the thicker absorber.

\subsection{Calorimeter BigCal}

The continuation of the recoil polarization measurements of the $G_{E p} / G_{M p}$ ratio to larger $\mathrm{Q}^{2}$ is possible only if the electron detector solid angle matches the spectrometer solid angle, i.e. all electrons associated with a proton detected in the HMS FPP, are detected; the reason is that the cross section has become quite small, $\sim 10^{-37} \mathrm{~cm}^{2}$. At $\mathrm{Q}^{2}=9 \mathrm{GeV}^{2}$ and with a beam energy of $6 \mathrm{GeV}$, this requires $\Delta \Omega_{e}=140 \mathrm{msr}$ (HMS has $7 \mathrm{msr}$ ). Such a solid angle matching can only be obtained with a large size calorimeter. We have constructed an electromagnetic calorimeter with 1744 bars of lead-glass, 1024 bars coming from Protvino and 720 bars from Yerevan; the individual bar size is $4 \times 4 \mathrm{~cm}^{2}$, length 45 and $40 \mathrm{~cm}$, respectively. The status of the calorimeter in late 2003 can be seen in Fig. 12 . The Čerenkov light produced by the showering of 1 to $2 \mathrm{GeV}$ electrons is detected, making the calorimeter insensitive to non-showering particles like protons and pions. The frontal area of the calorimeter is $2.8 \mathrm{~m}^{2}$.

\section{Other form factor measurements at JLab}

Two JLab experiments have measured the neutron electric form factor $G_{E n}$ in Hall C [16, 17], and one has measured the neutron magnetic form factor $G_{M n}$ in Hall B [20]. Two new experiments at JLab will measure the neutron electric form factor to larger $\mathrm{Q}^{2}$. The first will obtain the asymmetry in ${ }^{3} \overrightarrow{H e}(\vec{e} \rightarrow e n)^{2} H$ in Hall A to $Q^{2}=2.7 \mathrm{GeV}^{2}$ [18], and in the second the neutron recoil polarization in ${ }^{2} H\left(\vec{e} \rightarrow, e^{\prime} \vec{n}\right) p$ in Hall C to $\mathrm{Q}^{2}=4.3 \mathrm{GeV}^{2}[19]$ will be measured. With these two experiments we can expect that the characterization of the nucleon structure in elastic scattering will be much advanced in the near future. 


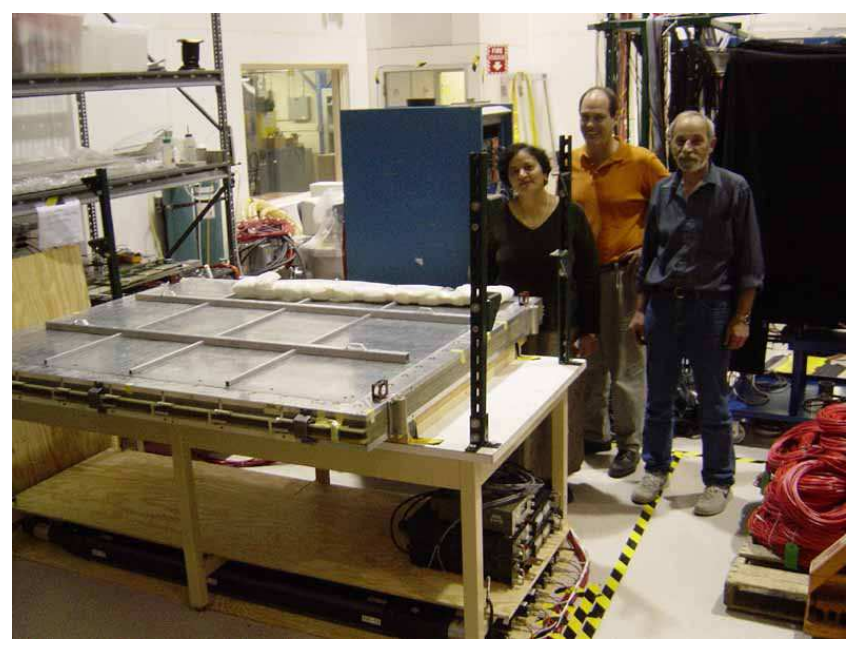

Figure 11: The prototype drift chamber built at the LHE is inspected by Drs. Vina Punjabi, Mark K. Jones and Lev Smykov at JLab, Nov. 2003.

\section{JLab upgrade to $12 \mathrm{GeV}$}

The JLab upgrade to an energy of $12 \mathrm{GeV}$ is now one of the top priorities for DOE. In the current scenario accelerator operation may be interrupted for 18 months sometimes in the time frame 2008-10, to install new arc magnets and new cavities. The $12 \mathrm{GeV}$ should become available for experiments in 2012. The $G_{E p} / G_{M p}$-ratio can be measured as soon as an energy larger than $8.5 \mathrm{GeV}$ becomes available in Hall $\mathrm{C}$, to a $\mathrm{Q}^{2}$ of 12 $\mathrm{GeV}^{2}$, using the new calorimeter and FPP being built for $G_{E p}(\mathrm{III})$ and the HMS. The recoil proton momentum will be $7.27 \mathrm{GeV} / \mathrm{c}$. A planned superHMS spectrometer would allow the measurement of the $G_{E p} / G_{M p}$-ratio to $14 \mathrm{GeV}^{2}$.

What will be $A_{y}$ ? We hope to have the answer from the anticipated second Dubna calibration. The linear extrapolation of the $A_{y}$ data from the first Dubna calibration [21], shown in Fig. 13 suggests that the analyzing power may be about $25 \%$ smaller than in the forthcoming $9 \mathrm{GeV}^{2}$ measurement; but we must check that nature behaves linearly with the planned next calibration at the Dubna Nuclotron.

\section{Conclusions}

We have presented the results of the two JLab recoil polarization experiments which measured the form factor ratio $G_{E p} / G_{M p}$ using the recoil polarization technique from 0.5 to $5.6 \mathrm{GeV}^{2}$. Above $\mathrm{Q}^{2}$ of about $2 \mathrm{GeV}^{2}$, these results are incompatible with ratios obtained using the traditional Rosenbluth separation method. This surprising finding has generated considerable work among theorists, but we do not have a definite explanation for the difference as of now; the two possibilities being discussed presently are the possibly incomplete radiate corrections and the previously neglected two-photon exchange contribution. Both affect the results of a Rosenbluth separation, but leave the recoil polarization essentially unchanged. 


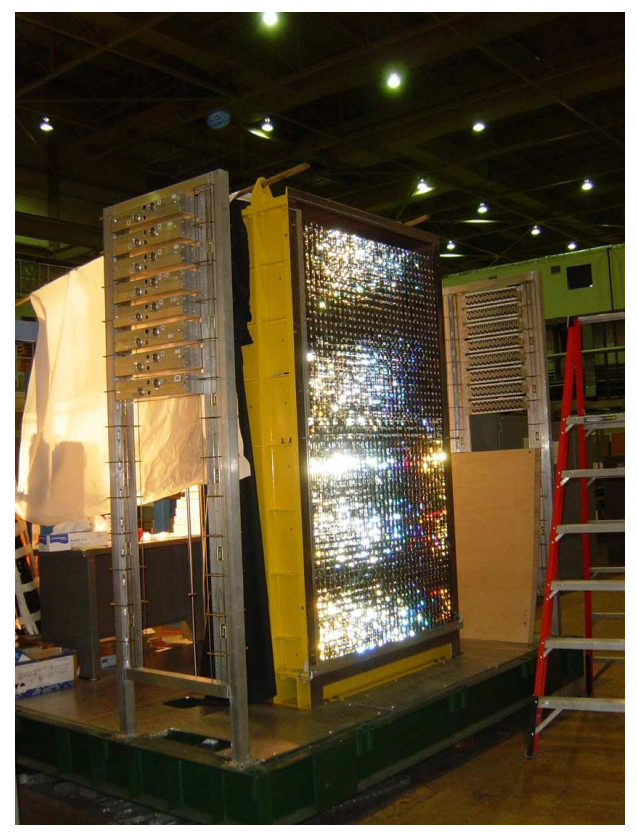

Figure 12: Front view of the calorimeter taken in November, 2003: all lead-glass bars have been stacked. Currently the calorimeter is fully assembled and is being tested by taking cosmics data.

The history of proton form factor measurements, which started with the pioneering work of R. Hofstadter 50 years ago, illustrates the danger of using only one method to measure a given physical observable; the Rosenbluth separation method has been for a long time the only technique available to separate the form factors of elastic, quasielastic and inelastic electron scattering on the nucleon and nuclei, as in $e p, A\left(e, e^{\prime} p\right)$ and $\left(e, e^{\prime} \Delta\right)$ or $\left(e, e^{\prime} N^{*}\right.$, respectively. A number of recent technological advances in accelerators and instrumentation, including high performing polarized beams and targets, and efficient polarimeters, have finally given us a second approach, and at the same time led to the discovery of a weakness of the older method, connected to the large radiative corrections required for cross section data.

The next measurement of the $G_{E p} / G_{M p}$ ratio, $\mathrm{GE}_{p}(\mathrm{III})$, is currently being prepared. It will extend the $\mathrm{Q}^{2}$ range to $9 \mathrm{GeV}^{2}$. There are several theoretical calculation which anticipate that the ratio might change sign at a $\mathrm{Q}^{2}$ smaller than $9 \mathrm{GeV}^{2}$. The two new data points this next experiment will provide, and their anticipated statistical uncertainties, are shown in Fig. 14.

With another analyzing power measurement in Dubna, the $\mathrm{Q}^{2}$ limit for $G_{E p} / G_{M p}$ can be pushed to $12 \mathrm{GeV}^{2}$ once the energy upgrade at JLab is completed. Such an experiment has been proposed [36] and it potentially could be one of the early experiments with the upgraded JLab accelerator.

New neutron form factor measurements are very important, as the nucleon must be understood in its two isospin state forms. In view of a future measurements of $G_{E n}$ there is also a need for neutron analyzing power data; the NUCLOTRON at JINR is the only facility in the World, where such measurements could be made in the near future. 


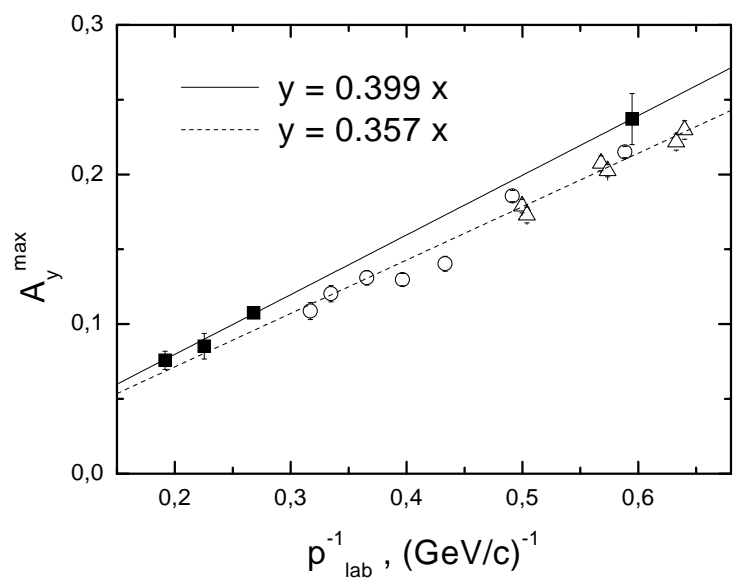

Figure 13: The maximum value of the analyzing power $A_{y}$ for carbon and $\mathrm{CH}_{2}$, versus the inverse of the proton momentum. The apparent linear behavior allows for a tentative extrapolation to larger momenta.

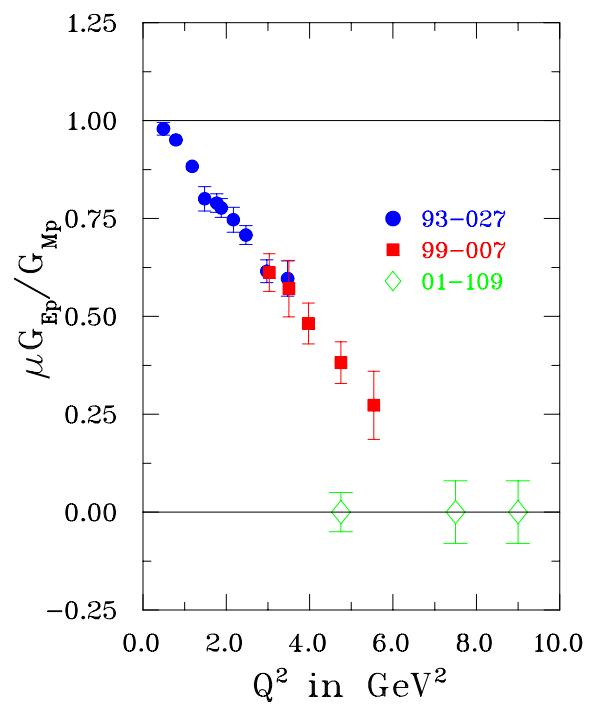

Figure 14: Results of the two previous JLab recoil polarization experiments, and the two new data points approved for expt. $04-108$.

This work was supported in part by the U.S. National Science Foundation (NSF PHY0140108) and the US Department of Energy (DEFG-02-89ER-40525). .

\section{References}

[1] M.K. Jones et al., Phys. Rev. Lett. 84, 1398 (2000).

[2] O. Gayou et al., Phys. Rev. Lett. 88, 092301 (2002).

[3] A.I. Akhiezer and M.P. Rekalo, Sov. Phys.-Doklady 13, 572 (1968).

[4] R. Arnold, C. Carlson and F. Gross, Phys. Rev. C23, 363 (1981).

[5] L. Andivahis et al., Phys. Rev. D 50, 5491 (1994).

[6] M.E. Christy et al., nucl-e/0401030.

[7] J. Arrington and R. Segel et al.,"New Measurements of $G_{E} / G_{M}$ for the Proton", JLab experiment 01-001.

[8] N. Merenkov, A. Afanasev and I. Akushevich, "Calculation of the QED correction to the recoil polarization by the electron structure function method", JLAB-THY-0003, and A.V. Afanasev, I. Akushevich, A Ilyichev and N. P. Merenkov, Phys. Lett. B 514, 269 (2001).

[9] E. Tomasi-Gustafsson and G.I. Gakh, hep-ph/0412137.

[10] P.A.M. Guichon and M. Vanderhaeghen, Phys. Rev. Lett. 91, 142303 (2003) 
[11] P.G. Blunden, W. Melnitchouk, J.A. Tjon, Phys. Rev. Lett. 91142304 (2003).

[12] Y.-C. Chen, A. Afanasev, S.J. Brodsky, C.E. Carlson and M. Vanderhaeghen, submitted to P.R.L., also arXiv:hep-ph/0403058.

[13] M.P. Rekalo and E. Tomasi-Gustafsson, Eur. Phys. J. A22, 331 (2004), and Nucl. Phys. A 740, 271 (2004), and Nucl. Phys. A 742, 322 (2004)

[14] C.F. Perdrisat, V. Punjabi, M.K. Jones and E. Brash, "Measurement of $G_{E p} / G_{M p}$ to $\mathrm{Q}^{2}=9 \mathrm{GeV}^{2}$ via recoil polarization" $\exp 04-108$.

[15] R. Gilman, L. Pentchev, C.F. Perdrisat and R. Suleiman, "Measurement of the Twophoton Exchange Contribution in ep Elastic Scattering using Recoil Polarization", exp.04-019.

[16] H. Zhu et al, Phys. Rev. Lett. 87, 081801 (2001).

[17] R. Madey et al Phys. Rev. Lett. 91, 122002 (2003).

[18] B. Reitz, B. Wojtsekhowski, K. McCormick, "Measurement of the Neutron Electric Form Factor $G_{E n}$ at High $\mathrm{Q}^{2}$, exp. 02-013.

[19] R. Madey, S. Kowalski, A. Yu. Semenov and B.D. Anderson, "The Neutron Electric Form Factor at $\mathrm{Q}^{2}=4.3 \mathrm{GeV}^{2}$ from the Reaction ${ }^{2} H\left(\vec{e}, e^{\prime} \vec{n}\right)^{1} H$ via Recoil Polarimetry", exp. 04-110.

[20] JLab proposal 94017, W. Brooks and M. Vineyard.

[21] L.S. Azhgirey et al, AIP Conf. Proc.675, 826 (2003) and to be published in NIM (2005).

[22] A.V. Belitsky, Xiandong Ji and Feng Yuan, hep-ph/0212351 v2 (2003).

[23] S.J. Brodsky, hep-ph/0208158 v1 (2002).

[24] F. Iachello, A.D. Jackson, and A. Landé, Phys. Lett. B 43, 191 (1973).

[25] G. Höhler et al., Nucl. Phys. B 114, 505 (1976).

[26] M.F. Gari and W. Kruempelmann, Z. Phys. A 322, 689 (1985) 689; Phys. Lett. B 274, 159 (1992); Phys. Lett. B 282, 483(E) (1992).

[27] G. Holzwarth, Zeitschr. Fuer Physik A 356, 339 (1996); G. Holzwarth, hepph/0201138 v1 (2002).

[28] M.R. Frank, B.K. Jennings and G.A. Miller, Phys. Rev. C 54, 920 (1996).

[29] F. Cardarelli and S. Simula Phys. Rev. C 62, 65201 (2000); S. Simula, e-print nuclth/0105024.

[30] E. Pace, G. Salme, F. Cardarelli and S. Simula, Nucl. Phys. A 666\&667, 33c (2000).

[31] E.L. Lomon, Phys.Rev. C 64, 035204 (2001). 
[32] B.-Q. Ma, D. Quing and I. Schmidt, Phys. Rev C 65035205 (2002).

[33] L.W. Mo and Y.S. Tsai, Rev. Mod. Phys. 41, 205 (1969).

[34] L.C. Maximon and J.A. Tjon, Phys. Rev. C 62, 054320 (2000).

[35] R. Suleiman, L. Pentchev, C.F. Perdrisat and R. Gilman, Jlab experiment 04-019.

[36] " $G_{E p} / G_{M p}$ with an $11 \mathrm{GeV}$ electron beam"; C.F. Perdrisat, V. Punjabi, M.K. Jones and E.J. Brash, letter of intent in $12 \mathrm{GeV}$ proposal CD0, (2000), JLab. 